\title{
Inserção em grupos formais e qualidade de vida entre adolescentes
}

\author{
Cheila de Oliveira Bueno ${ }^{1}$ - Universidade Luterana do Brasil, Canoas, Brasil \\ Miriam Raquel Wachholz Strelhow - Universidade Luterana do Brasil, Canoas, Brasil \\ Sheila Gonçalves Câmara - Universidade Luterana do Brasil, Canoas, Brasil
}

\begin{abstract}
Resumo
Este estudo avalia a relação entre afiliação a grupos formais e sexo e autoestima, satisfação com a vida e propósito de vida, conjuntamente, enquanto componentes da qualidade de vida de adolescentes. Trata-se de um estudo transversal, com uma amostra de 748 estudantes do $1^{\circ}$ ano do ensino médio, com idades entre 13 e 22 anos, alunos de escolas públicas estaduais de um município da grande Porto Alegre. Os instrumentos utilizados para coleta de dados foram: Pil Test, Brief Life Satisfaction Scale e Escala de Auto-Estima de Rosenberg. Os resultados apontam que as três dimensões avaliadas, conjuntamente, foram significativamente afetadas pela pertença a grupos $(\mathrm{F}(3,698)=2,88, \mathrm{p}<0,05)$, mas não por sexo. A qualidade de vida adolescente é melhor entre os jovens que participam de grupos formais. $\mathrm{O}$ estudo aponta para a participação em grupos formais como um indicativo de saúde positiva e um aspecto relevante para a promoção da saúde.

Palavras-Chave: Adolescência, Pertença a grupos formais, Qualidade de vida.
\end{abstract}

Insertion in formal groups and quality of life among adolescents

\begin{abstract}
This present study evaluates the relation between affiliation to formal groups and gender and self-esteem, life satisfaction and purpose of life, altogether, as components of the quality of life of adolescents. This is a transversal study, with a sample of 748 teenager students from the first year of high school, ages between 13 and 22 years-old, of state public schools of a city in the metropolitan area of Porto Alegre. The instruments used to collect the data were: Pil Test, Brief Life Satisfaction Scale and Rosenberg's Self-Esteem Scale. The results indicate that the three aspects evaluated, altogether, were significantly affected by the affiliation to groups $(F(3,698)=2,88, p<0,05)$, but not by gender. The adolescent quality of life is better among youngsters who take part in formal groups. The study points out to the participation in formal groups as an indicative of positive health and a relevant aspect for health promotion.

Keywords: Adolescence, Affiliation to formal groups, Quality of life.
\end{abstract}

A Organização Mundial de Saúde (OMS) define a adolescência como o período compreendido entre os 10 e 19 anos. Porém, estudos indicam que a puberdade tem início cada vez mais cedo, e o ingresso na vida adulta tem sido adiado por diversos fatores sociais, como o aumento do período de formação escolar, a alta competitividade do mercado de trabalho, poucas ofertas de empregos e a dificuldade em tornar-se independente financeiramente (Kell, 2004; Heilborn, 2006). Em face disso, autores como Klosinski (2006) defendem a extensão do período da adolescência, definindo-o de 10 aos 25 anos.

O termo juventude refere-se a uma categoria sociocultural e historicamente construída a partir de critérios múltiplos, que englobam as dimensões biopsicológica, cronológica e social. Não deve ser concebida, portanto, como um conjunto de fenômenos universais, pois as transformações pelas quais passam os jovens também resultam de processos inerentes aos contextos sociais - históricos, políticos e econômicos nos quais os indivíduos estão inseridos (Ferreira, Alvinir, Teixeira \& Veloso, 2007). Como forma de

${ }^{1}$ Endereço para correspondência:

Rua Alberto Pasqualine, n 915 - Bairro Sumaré - Alvorada/RS

E-mail: cheobueno@yahoo.com.br melhor denominar essa população, que ultrapassa uma definição cronológica, no presente estudo são utilizados ambos os termos - adolescente e jovem, pois se entende que ambas as nominações conseguem definir a população estudada.

A influência dos aspectos sociais mais abrangentes manifesta-se nas relações mais próximas estabelecidas pelo adolescente com as pessoas que the são importantes. Os grupos sociais revelam-se como preponderantes nessa etapa da vida em termos da definição de normas e valores que contribuem para a construção da identidade pessoal. Isso envolve os processos de identificação e diferenciação em relação ao grupo (Bulgacov, Ribeiro, Cobalchini, Souza \& Diório, 2001).

Há uma busca pela separação gradual da família e direcionamento para o grupo de pares. Surgem relações interpessoais cada vez mais aprofundadas fora do contexto exclusivamente familiar, mediante a aproximação com iguais ou mesmo outros adultos significativos (Montemayor, 1994; Romanelli \& Prieto, 2002). Assim, a formação dos grupos de pares torna-se mais preponderante nessa fase, e adquire um papel transitório entre o distanciamento da família de origem e a formação de novo grupo familiar (Heilborn, 2006). 
A tendência dos adolescentes a agrupar-se proporciona novas experiências e pode dar origem a grupos de cunho cultural, religioso e esportivo, bem como às tribos urbanas (Pavía, Gerlero \& Apendino, 1995). Esses grupos formam subculturas, identificadas com diferentes estilos, muitas vezes impulsionadas por preferências estéticas e musicais, mas também fortemente relacionadas à realidade social que as circunda (Feixa, 1988). Atualmente, com o estabelecimento definitivo da internet e a ampliação do acesso, esta tornou-se o meio de comunicação preferido dos adolescentes. Eles podem desempenhar diferentes papéis, protegidos, muitas vezes, pelo anonimato, bem como estabelecer relações com pessoas que partilham as mesmas escolhas, trocando informações e impressões sobre temas de seu interesse (Abrantes, 2002). Isso amplia em muito a possibilidade de inserção em grupos, que até o advento da internet, eram apenas os grupos reais e concretos.

Podem-se definir os grupos sociais dos quais os adolescentes participam em dois tipos: grupos formais e informais. No primeiro estão os grupos oficiais, com patentes (formalmente estabelecidos), objetivos definidos e encontros regulares, aptos a propiciar mais estrutura, com normas fortes e maiores expectativas de seus membros, como, por exemplo, grupos de escoteiros, companhias de dança, ou grupos de jovens vinculados a instituições religiosas. Os grupos informais são menos bem definidos, baseados na amizade, organização de estudo ou hobbies/ interesses casuais. Assim, os membros de grupos formais tendem a sentir menos autonomia pessoal em seus grupos, mas também maior clareza acerca de seu grupo comparado a outros (Sheldon \& Bettencourt, 2002).

Fatores sociais, como classe, religião, raça e outros, referentes ao prestígio da pessoa no grupo determinam, em grande parte, os valores acerca de si mesmo que, por sua vez, exercem influência sobre a autoestima (Rosenberg, 1986). A autoestima relacionase a qualquer valor a que o grupo no qual o indivíduo está inserido atribua importância (Sarriera, Schwarcz \& Câmara, 1996).

Para Sánchez e Escribano (1999), a representação social que o adolescente tem de si mesmo está embasada tanto no autoconceito quanto na autoestima. São atribuições individuais aperfeiçoadas nas relações cotidianas desde a infância e que agem como fatores decisivos na relação do indivíduo consigo mesmo e com os outros.

Tais relações melhor configuram o bem-estar, que pode favorecer a maneira como as pessoas veem a si e aos outros, resultando em maior prazer na vivência das situações do dia a dia e no relacionamento com os pares (Passareli \& Silva, 2007). A sensação de bem- estar ou de satisfação com a vida está ligada à forma como o indivíduo é capaz de lidar e absorver os episódios de sua vida (Silva \& colaboradores, 2007).

Costa e Bigras (2007) afirmam que as questões de saúde e bem-estar na adolescência e na infância devem ter um enfoque diferenciado em relação à população adulta, considerando os determinantes sociais (sistemas de saúde, educação, trabalho, desenvolvimento social, direitos necessários à integração social das famílias), espaços de convivência, formação e intervenção; bem como os determinantes familiares influenciados pelo ambiente sociocultural. $\mathrm{Na}$ adolescência, a satisfação com a vida está relacionada a aspectos comunitários e econômicos, relações familiares, experiências escolares e relações com pares (Barros, Gropo, Petribú \& Colares, 2008).

Como um período de reestruturação identitária, a adolescência permite o resgate da história de vida até o momento e abre-se na perspectiva de escolhas para a vida futura, que se configuram como um propósito de vida. As perspectivas quanto ao tempo futuro consistem no estabelecimento de metas de vida, também denominadas de tarefas de vida ou projetos. $\mathrm{Na}$ busca da realização dessa exigência pessoal e psicossocial, os adolescentes recebem influências de seus grupos de referência, que podem representar tanto contribuições positivas como fatores de maior indecisão (Locatelli, Bzuneck \& Guimarães, 2007).

O estabelecimento de um projeto é uma tarefa determinada socioculturalmente, determinada pelas contingências de vida do indivíduo. Espera-se que, no final da adolescência, como ingrediente da formação de sua identidade pessoal, as pessoas tenham um direcionamento quanto ao seu futuro. Estudos sobre projetos de vida de adolescentes demonstram que a educação, o trabalho e a constituição de família representam os organizadores centrais das representações sociais do futuro presentes em suas metas vitais (Nascimento, 2006; Paredes \& Pecora, 2004).

Estudos sobre a adolescência apontam para diferenças significativas entre os meninos e as meninas no que tange a papéis sociais, projetos de vida e fatores relacionados ao bem-estar (Garaigordobil, 2009; Goldbeck, Schmitz, Besier, Herschbach \& Henrich, 2007; Schoen-Ferreira, Aznar-Farias \& Silvares, 2009). Bassen e Lamb (2006) avaliaram diferenças entre os sexos e as mudanças ocorridas no desenvolvimento na autopercepção de adolescentes acerca de sua afiliação, afiliação negativa e assertividade. Os resultados apontaram que as meninas se sentiam com mais afiliação e menos afiliação negativa em seus papéis que os meninos, enquanto as diferenças na assertividade não foram significativas. Os autores apontam pequenas 
diferenças entre os sexos nas habilidades cognitivas e psicossociais, estas tendo diminuído no decorrer das últimas décadas, e que as diferenças crescem em alguns contextos e situações, mas não em outros.

Em todas as etapas da vida, mas especialmente na adolescência, a rede de apoio social é aquela que dá suporte para o enfrentamento das mudanças decorrentes do ciclo vital ou outros estressores, ajuda a estruturar a interpretação dos fenômenos presentes no contexto de vida da pessoa e possibilita o direcionamento para o futuro. Em face disso, o presente estudo visa: a) avaliar a relação existente entre afiliação a grupos formais e as dimensões de autoestima, de satisfação com a vida e de propósito de vida; e b) avaliar a relação existente entre o sexo e as dimensões de autoestima, de satisfação com a vida e de propósito de vida entre adolescentes escolares.

\section{Método}

O presente estudo observacional, analítico de corte transversal foi realizado com estudantes de primeiro ano do ensino médio da rede pública estadual de Alvorada/RS. Alvorada é um município da Região Metropolitana de Porto Alegre, com cerca de 214 mil habitantes, cujas principais atividades econômicas são comércio e serviços (IBGE, 2009). De acordo com os dados da Secretaria da Educação do Rio Grande do Sul (SEC/RS, 2009), em 2008, havia 1.286 alunos matriculados no primeiro ano do ensino médio, no turno diurno. Foi selecionado esse turno de estudo por nele haver um perfil de adolescentes com maior disponibilidade para participar de grupos formais, o que se diferencia do perfil dos estudantes noturnos, que têm faixa etária mais elevada e, geralmente, trabalham. Nesse sentido, a faixa etária prevista era dos 13 aos 18 anos. No entanto, 11 jovens $(1,6 \%)$ tinham idade entre 19 e 22 anos. Optou-se por mantê-los no estudo em razão de sua inserção em grupos formais.

Utilizou-se o programa Epi-Info para os cálculos de tamanho de amostra, considerando-se o total de escolares matriculados, uma prevalência esperada do desfecho de $50 \%$ e um erro máximo tolerado de $\pm 3 \%$. Com isso, a amostra inicialmente calculada foi de 583 jovens. Considerando-se um possível efeito de delineamento de 1,2, essa amostra foi ampliada em 116 sujeitos, acrescidos de mais 15\% para suportar uma perda estimada de $15 \%$. Com isso, o tamanho da amostra foi estimado em 786 jovens. A amostra final constituiu-se de 748 adolescentes estudantes de seis escolas.

Como instrumentos, foram utilizados: um questionário de dados sociodemográficos (sexo, idade, grau de escolaridade dos pais) e um questionário sobre afiliação grupal (participação e frequência), ambos desenvolvidos especificamente para este estudo com base na literatura sobre o tema (Bulgacov \& colaboradores, 2001; Sheldon \& Bettencourt, 2002). Dessa forma, a questão sobre a participação em grupos constava de oito categorias (não participo de nenhum grupo, grupo de dança, grupo de música, grupo de escoteiros, grupo de jovens - religioso, grupo políticopartidário, grupo de prática de esporte formal, outro. Qual?). As respostas à categoria outros puderam ser inseridas dentro das categorias previamente estabelecidas.

Para avaliar o propósito de vida foi utilizado o PIL - test (Purpose in Life). Este, desenvolvido originalmente por Crumbaugh (Baiocchi \& Magalhães, 2004), tem como base a Psicologia Existencial de Frankl (1987). Para este estudo foi utilizada a versão revisada feita por Harlow, Newbomb e Bentler (1987), o PIL-R. O instrumento é composto de 20 itens referentes à capacidade de encontrar sentido e motivação para a vida. As respostas são assinaladas numa escala tipo Likert de sete pontos, indicando graus diferentes de concordância com as afirmações. No Brasil, o instrumento foi utilizado em estudo como os de Baiocchi e Magalhães (2004) com profissionais graduados $(\mathrm{alfa}=0,90)$, e Aquino e colaboradores (2010) com universitários (alfa $=0,81$ ). No presente estudo, a escala obteve um alfa de 0,78.

Para a avaliação da satisfação com a vida foi utilizada a Brief Multidimensional Students' Life Satisfaction Scale (BMSLSS: Huebner, Suldo, Valois, Darne \& Zullig, 2004). A BMSLSS é uma versão breve da Multidimensional Students' Life Satisfaction Scale (MSLSS: Huebner, Laughlin, Ash \& Gilman, 1998). A BMSLSS foi desenvolvida para ser uma escala autoaplicável breve. Consiste em uma medida de cinco itens (satisfação com a família, amigos, experiência como estudante, consigo mesmo e com o lugar onde vive), acrescida de um item sobre a satisfação com a vida em geral, a serem respondidos em escala Likert de 11 pontos, com opções que variam de "péssima" a "excelente". A soma dos resultados dos sujeitos nos seis itens fornece um escore de satisfação geral com a vida. Para o presente estudo, a BMSLSS foi traduzida diretamente da versão original em inglês, que é bastante simples (todos os itens apresentam a mesma estrutura: "descreveria minha satisfação com... como...". Os domínios família, amigos/as, experiência como estudante, comigo mesmo/a, lugar onde vivo e minha vida em geral dão o sentido a cada item). A versão obtida foi submetida à análise semântica mediante a aplicação do instrumento a seis adolescentes com idades entre 13 e 15 anos, não tendo sido necessária nenhuma reformulação dos itens. Em estudo piloto 
com 188 adolescentes estudantes de sétima e oitava séries de escolas estaduais do município de Cachoeirinha/RS, a escala obteve um índice alfa de Cronbach de 0,92 com os seis itens e 0,90 com os cinco itens referentes a domínios específicos. No presente estudo a escala com seis itens obteve um alfa de 0,82 e com cinco itens 0,75 .

A autoestima dos jovens foi avaliada através da Escala de Autoestima de Rosenberg (1986). Esta foi desenvolvida originalmente para medir sentimentos globais de adolescentes em relação a autovalor ou autoaceitação e é geralmente considerada a medida padrão em comparação a outras medidas de autoestima. A escala inclui 10 itens que são pontuados utilizando um agrupamento de respostas de quatro pontos que vão de discordo fortemente a concordo fortemente (Adler \& Stewart, 2004). A versão utilizada no estudo foi a proposta por Assis e colaboradores (2003) que, em estudo com 1685 estudantes de São Gonçalo/RJ encontraram, no pré-teste do instrumento um alfa de Cronbach de 0,68. Neste estudo o instrumento apresentou um alfa de 0,65.

Para a coleta de dados verificou-se, junto à Secretaria de Educação do Rio Grande do Sul (SECRS, 2009), que havia 11 escolas estaduais com ensino médio regular no município de Alvorada/RS. Destas, seis foram sorteadas aleatoriamente e contatadas a fim de que fornecessem sua autorização para participar do estudo. Realizada essa etapa, os alunos de primeiro ano do ensino médio foram convidados a participar da pesquisa, com a devida explicação dos objetivos da mesma. Os participantes responderam ao instrumento de pesquisa mediante sua autorização prévia (assinatura do Termo de Consentimento Livre e Esclarecido TCLE), no caso dos maiores de idade, ou de seus pais ou responsáveis, para os menores de idade, em conformidade com a Resolução 196 do Conselho Nacional de Saúde (CNS), no que diz respeito à pesquisa com seres humanos (Ministério da Saúde, 1997). Como medida para evitar as perdas com relação ao consentimento dos pais, houve comunicação prévia das escolas sobre a pesquisa em reuniões de pais. Além disso, a entrega dos TCLE era feita no dia anterior à aplicação dos instrumentos. Em cada escola, as pesquisadoras voltaram até três vezes para realizar aplicação entre os que não haviam trazido o TCLE assinado, sempre com nova entrega dos termos no dia anterior às aplicações. Aqueles jovens que após as três tentativas não trouxeram o TCLE assinado foram considerados como perdas. $\mathrm{O}$ instrumento foi aplicado de forma grupal pelas pesquisadoras nas salas de aula (primeira aplicação) ou em sala específica na segunda e terceira aplicações), com um tempo médio de 30 minutos.
Os dados foram analisados por meio de estatística univariada (frequências e porcentagens, médias e desvio padrão) para a descrição dos sujeitos e seu envolvimento em grupos; estatística bivariada, pelo teste Qui-Quadrado para avaliar as diferenças entre os sexos e faixas etárias quanto à participação em grupos formais; bem como o teste $t$ de Student para avaliar as diferenças entre os grupos conforme as variáveis sexo, faixa etária e participação em grupos formais em relação às variáveis autoestima, propósito de vida e satisfação geral. Foi realizada análise multivariada de variância (MANOVA) entre sujeitos para avaliar o efeito da interação dos fatores em estudo pertença a grupos formais (não e sim) e sexo (feminino e masculino) com o conjunto das variáveis dependentes (propósito de vida, autoestima e satisfação com a vida).

\section{Resultados}

Dentre os jovens que responderam aos instrumentos, foram excluídos 43 , dos quais 38 por respostas omissas em itens dos instrumentos que avaliavam propósito de vida, autoestima e satisfação com a vida e cinco na variável sexo. Dos 705 sujeitos restantes, $383(54,3 \%)$ eram do sexo feminino e a idade variou de 13 a 22 anos $(M=15,30, D P=1,22)$.

Quanto ao nível de estudos dos pais, considerando os sujeitos que responderam a esta questão, no que tange às mães, 34,6\% tinham ensino fundamental completo, $27,9 \%$ ensino médio completo, $16,8 \%$ ensino fundamental incompleto, $12,8 \%$ ensino médio incompleto, $6,3 \%$ curso superior e $1,5 \%$ não tinha estudos. Dentre os pais, $31,7 \%$ tinham ensino fundamental incompleto, $26,6 \%$ ensino médio completo, $15,7 \%$ ensino médio incompleto, $14,5 \%$ ensino fundamental completo, $8,6 \%$ curso superior e $2,8 \%$ não tinham estudos.

Em relação à pertença a grupos formais, 43,4\% pertenciam a algum grupo formal. Destes, 50,3\% participam de grupos de prática de esporte, $27,1 \%$ de grupos de jovens (religioso), 17\% de grupos de dança, $15,7 \%$ de grupos de música, $2,6 \%$ de grupos de escoteiros e $2,3 \%$ de grupos políticos. Sobre o tempo que os jovens participam dos referidos grupos, verificase a predominância de participação por um ano ou mais: $61 \%$ nos grupos esportivos, $70,1 \%$ nos grupos de jovens, $60 \%$ nos grupos de dança, $65,3 \%$ nos grupos musicais, $87,5 \%$ nos grupos de escoteiros e $50 \%$ nos grupos políticos. A frequência de participação que predomina é de uma a mais vezes por semana $(68,7 \%$ : grupos esportivos, 81,6\%: grupos de jovens, $77,7 \%$ : grupos de dança, 57,2\%: grupos musicais, 87,5\%: grupos de escoteiros e $50 \%$ : grupos políticos). Quanto à motivação para a participação nos grupos, o desejo 
pessoal (escolha do jovem) aparece como prioritário (85,2\%: grupos esportivos, $85 \%$ : grupos de jovens, 56,7\%: grupos de dança, 90,8\%: grupos musicais, 100\%: grupos de escoteiros e 100\%: grupos políticos).

Ao avaliar a distribuição dos sexos por inserção em grupos formais, verificou-se que houve diferença significativa na participação de grupos de dança $\left(\mathrm{X}^{2}=15,59 ; \mathrm{p}<0,01\right)$ com $78,3 \%$ de participação das meninas, grupos de jovens $\left(\mathrm{X}^{2}=7,60 ; \mathrm{p}<0,05\right)$ com $67,8 \%$ com participação das meninas e grupos de prática de esporte $\left(\mathrm{X}^{2}=50,07 ; \mathrm{p}<0,01\right)$, com $70,1 \%$ de participação dos meninos.

Considerando a divisão do grupo em duas faixas etárias (de 13 a 18 anos e de 19 a 22 anos) em relação à participação em grupos formais, verificou-se que não houve diferenças significativas entre os sujeitos com idade até 18 anos e os de 19 a 22 anos, embora tenha sido possível verificar uma maior participação dos jovens com idades entre 19 e 22 anos em grupos formais de práticas de esportes $(45,5 \%$ dos jovens desta faixa etária).

Em relação aos sexos, não houve diferença significativa em nenhuma das três variáveis psicológicas em estudo, da mesma forma que à faixa etária. Quanto à participação em grupos formais em relação a suas médias nas variáveis autoestima, propósito de vida e satisfação com a vida, os resultados demonstram que houve diferenças estatisticamente significativas entre os grupos nas três variáveis avaliadas, apresentando, os adolescentes que pertencem a grupos formais, maior autoestima, propósito de vida e satisfação com a vida (Tabela 1).

Tabela 1 - Diferença nas médias entre grupos por sexo, faixa etária e participação em grupos nas variáveis psicológicas

\begin{tabular}{|c|c|c|c|c|c|}
\hline Variáveis psicológicas & & $\mathrm{M}$ & DP & $\overline{\mathrm{T}}$ & $p$ \\
\hline \multicolumn{6}{|c|}{ Sexo } \\
\hline \multirow[t]{2}{*}{ Autoestima } & Feminino & 2,34 & 0,67 & 1,01 & 0,311 \\
\hline & Masculino & 2,29 & 0,60 & & \\
\hline \multirow[t]{2}{*}{ Propósito de vida } & Feminino & 5,21 & 0,89 & $-0,25$ & 0,801 \\
\hline & Masculino & 5,23 & 0,81 & & \\
\hline \multirow[t]{2}{*}{ Satisfação geral } & Feminino & 7,54 & 1,60 & $-1,60$ & 0,110 \\
\hline & Masculino & 7,74 & 1,77 & & \\
\hline & Idade & & & & \\
\hline \multirow[t]{2}{*}{ Autoestima } & 13-18 anos & 2,31 & 0,63 & $-0,73$ & 0,87 \\
\hline & 19-22 anos & 2,34 & 0,64 & & \\
\hline \multirow[t]{2}{*}{ Propósito de vida } & 13-18 anos & 5,21 & 0,85 & $-1,08$ & 0,48 \\
\hline & 19-22 anos & 5,35 & 0,62 & & \\
\hline \multirow[t]{2}{*}{ Satisfação geral } & 13-18 anos & 7,62 & 1,67 & $-0,16$ & 0,30 \\
\hline & 19-22 anos & 8,07 & 1,36 & & \\
\hline & rtença a grup & & & & \\
\hline \multirow[t]{2}{*}{ Autoestima } & $\operatorname{Sim}$ & 2,24 & 0,57 & $-2,41$ & 0,016 \\
\hline & Não & 2,36 & 0,67 & & \\
\hline \multirow[t]{2}{*}{ Propósito de vida } & Sim & 5,30 & 0,84 & 2,33 & 0,020 \\
\hline & Não & 5,15 & 0,85 & & \\
\hline \multirow[t]{2}{*}{ Satisfação geral } & Sim & 7,82 & 1,63 & 2,68 & 0,007 \\
\hline & Não & 7,49 & 1,70 & & \\
\hline
\end{tabular}

O teste de MANOVA revelou que as variáveis dependentes (propósito de vida, autoestima e satisfação com a vida), em conjunto, foram significativamente afetadas pela pertença a grupos formais $[\mathrm{F}(3$, $698)=2,88, p<0,05]$. Foram encontradas diferenças univariadas significativas nas três variáveis dependentes, em todas elas os jovens que participavam de grupos formais apresentando médias mais elevadas, o que indica maior clareza de propósito de vida $[\mathrm{F}(1,700)=6,47, \mathrm{p}<0,05]$, maior autoestima $[\mathrm{F}(1$, $700)=4,44, p<0,05]$ e maior satisfação com a vida $[\mathrm{F}(1,700)=5,50, \mathrm{p}<0,05]$. O teste de MANOVA não revelou efeito global significativo de sexo, nem de interação entre sexo e pertença a grupos (Tabela 2). 
Tabela 2 - Médias e desvios padrão das três variáveis independentes por pertença a grupos e sexo (Alvorada/RS, 2009) $(\mathrm{n}=748)$

\begin{tabular}{|c|c|c|c|c|c|c|c|}
\hline \multicolumn{8}{|c|}{ Pertença a grupos formais } \\
\hline \multirow[b]{2}{*}{ Medidas } & \multicolumn{2}{|c|}{$\underset{(n=306)}{\operatorname{Sim}}$} & \multicolumn{2}{|c|}{$\begin{array}{c}\text { Não } \\
(\mathrm{n}=399)\end{array}$} & \multirow[b]{2}{*}{$\mathrm{F}$} & \multirow[b]{2}{*}{ df } & \multirow[b]{2}{*}{$\mathrm{p}$} \\
\hline & $\mathrm{M}$ & $\mathrm{DP}$ & M & $\mathrm{DP}$ & & & \\
\hline Propósito de vida & 5,31 & 0,85 & 5,15 & 0,86 & 6,47 & 1 & 0,01 \\
\hline Autoestima & 3,90 & 0,69 & 3,78 & 0,78 & 4,44 & 1 & 0,03 \\
\hline \multirow{3}{*}{$\begin{array}{l}\text { Satisfação com a vida } \\
F=2,88 ; D F=3,698 ; p=0,03\end{array}$} & 7,81 & 1,65 & 7,50 & 1,69 & 5,50 & 1 & 0,01 \\
\hline & \multicolumn{4}{|c|}{ Sexo } & & & \\
\hline & \multicolumn{2}{|c|}{$\begin{array}{l}\text { Masculino } \\
(\mathrm{n}=322)\end{array}$} & \multicolumn{2}{|c|}{$\begin{array}{l}\text { Feminino } \\
(\mathrm{n}=383)\end{array}$} & & & \\
\hline Medidas & $\mathrm{M}$ & $\mathrm{DP}$ & $\mathrm{M}$ & $\mathrm{DP}$ & $\mathrm{F}$ & df & $\mathrm{p}$ \\
\hline Propósito de vida & 5,23 & 0,81 & 5,21 & 0,89 & 0,09 & 1 & 0,76 \\
\hline Autoestima & 3,86 & 0,71 & 3,80 & 0,77 & 0,92 & 1 & 0,33 \\
\hline $\begin{array}{l}\text { Satisfação com a vida } \\
F=0,91 ; D F=3,698 ; p=0,43\end{array}$ & 7,75 & 1,77 & 7,53 & 1,60 & 2,47 & 1 & 0,11 \\
\hline
\end{tabular}

\section{Discussão}

Os resultados apontam uma participação de $43,4 \%$ dos jovens em grupos formais, em que destes os que têm maior participação são os grupos de prática de esportes $(50,3 \%)$ e os grupos de jovens - religiosos $(27,1 \%)$. Quando questionados sobre a motivação para participarem nos grupos, os jovens afirmam como prioritário o desejo próprio e indicam uma participação de uma vez por semana ou mais, indo ao encontro da literatura que aponta para a necessidade dos jovens estarem envolvidos com grupos de pares e, no caso deste estudo, grupos formais, especificamente (Heilborn, 2006; Romanelli \& Prieto, 2002).

Ao avaliar a distribuição dos sexos por inserção em grupos formais, verificou-se maior participação das meninas em grupos de dança e nos grupos de jovens, enquanto os meninos têm maior participação nos de prática de esporte. Porém não houve diferença significativa em relação às faixas etárias, o que aponta que, mesmo após os 18 anos, os jovens continuam participando de grupos formais, ainda que a literatura demonstre que há uma diminuição da necessidade dos jovens de estarem com seus pares à medida em que se aproximam da idade adulta (Heilborn, 2006; Romanelli \& Prieto, 2002).

Este artigo se propôs a avaliar a relação entre a participação dos adolescentes em grupos formais com propósito de vida, autoestima e satisfação com a vida. Constatou-se que todos os aspectos estudados, em interação, relacionaram-se positivamente à participação em grupos. Considerando-se os pressupostos da Psicologia Positiva, os resultados, além de revelarem a importância dos grupos estruturados para uma melhor qualidade de vida dos jovens, demonstram a interrelação entre os construtos estudados como uma dimensão positiva da vida.

A ideia de integração de construtos positivos também foi sugerida por Ryff (1989), que propôs, a partir de uma análise da literatura, um modelo teórico de bem-estar psicológico formado por seis dimensões: autoaceitação, relações positivas com os outros, autonomia, domínio do ambiente, propósito de vida e crescimento pessoal. A autora sugeriu que, dentre as dimensões avaliadas, um componente crítico da saúde mental é a crença que dá ao indivíduo o sentimento de que há um propósito e um significado para a vida, e quem tem atitudes positivas tem metas, intenções e um senso de direção, o que contribui para o sentimento de que a vida tem um sentido pleno. Dessa forma, um alto escore de propósito de vida contribui para o estabelecimento de objetivos e o sentimento de que a vida tem um sentido pleno. Esse construto faz referência aos processos básicos de motivação inerentes à vida humana, estando na busca por um sentido para a vida, que se torna importante especialmente em momentos de adversidade (Baiochhi \& Magalhães, 2004).

No presente estudo, a participação dos adolescentes em grupos associou-se à qualidade de vida, sendo o propósito de vida um dos fatores mais relevantes para sua formação. Corroborando este resultado, Yalom (1984) propôs que um sentido positivo de vida está ligado fortemente a crenças religiosas, dedicação a uma causa e metas claras, e participação em grupo. Adler (1997) aponta a importância do propósito de vida relacionado à saúde, descrevendo estudos em que o propósito de vida correlacionou-se negativamente com relatos de sofrimento e com sintomas psicológicos negativos; 
mediou a relação entre estresse incontrolável e uso de substâncias, e teve efeitos consistentes e diretos no bem-estar.

É possível afirmar que os grupos formais funcionam para os jovens pesquisados como fonte de suporte social que, segundo Danielsen, Samdal, Hetland e Wold (2009), pode ser definido como processos de troca social que contribuem para o desenvolvimento de padrões de comportamento, cognições sociais e valores. Para os autores existem consideráveis evidências de que o suporte social da família, de professores e pares está associado com a satisfação de vida percebida.

Em relação à satisfação com a vida em geral, o presente estudo aponta para melhores índices nos jovens que participam de grupos formais. Barros e colaboradores (2008) afirmaram que em 19\% dos estudos encontrados na literatura, a qualidade de vida é definida como satisfação com a vida e consigo mesmo. Os demais estudos incluem medidas de qualidade de vida como felicidade, bem-estar, desempenho, funcionamento, realização de metas, necessidade de satisfação e saúde. Nesse sentido, os resultados encontrados no presente estudo revelam que a participação em grupos formais representa um importante fator de qualidade de vida entre adolescentes.

Não foram identificadas diferenças significativas na relação entre sexo e os construtos de autoestima, satisfação com a vida e propósito de vida, diferente do estudo de Goldbeck e colaboradores (2007), que analisaram os efeitos do sexo na satisfação com a vida de 1.274 adolescentes, com idades entre 11 e 16 anos. Os resultados apontaram que as meninas relataram índices significativamente mais baixos de satisfação com a vida em geral e relacionada à saúde. Em ambos os sexos e em quase todos os domínios da vida, houve uma significativa diminuição da satisfação com a vida em geral.

Ao avaliar a relação de pertença a grupos e autoestima, identificou-se que os adolescentes que pertencem a grupos formais apresentam melhor autoestima que os demais. Quanto ao sexo, não houve diferença significativa, diferenciando-se do estudo de Antunes e colaboradores (2006), que encontraram diferenças significativas entre os sexos. Para os autores há uma tendência de que meninos apresentem melhores médias de autoestima do que as meninas.

A autoestima apresentou-se como um construto explicativo positivo para a diferença entre quem pertence e quem não pertence a grupos. Para Costa (2000), a autoestima talvez seja a variável mais crítica que afeta a participação exitosa de um adolescente com outros em um projeto, pois os adolescentes com menor autoestima têm maior dificuldade com a integração grupal e desenvolvem mecanismos que provavelmente distorcem a comunicação de seus pensamentos e sentimentos, dificultando essa integração.

\section{Considerações Finais}

Outros estudos (Antunes \& colaboradores, 2006; Baiocchi \& Magalhães, 2004; Barros \& colaboradores, 2008; Borges \& Argolo, 2002; Passareli \& Silva, 2007) já foram realizados apontando para a interação das dimensões satisfação com a vida, propósito de vida e autoestima entre si e de sua correlação com fatores sociodemográficos (idade, sexo, escolaridade, profissão), não tendo sido encontrados estudos que relacionassem diretamente tais construtos à participação em grupos formais.

Este estudo avaliou tal relação, identificando que as três dimensões positivas avaliadas indicam que, para esta população, a participação em grupos formais é um fator positivo para a saúde psicológica e, consequentemente, para a saúde geral. $\mathrm{O}$ fato de que as dimensões avaliadas identificam diferenças entre os jovens que participam ou não de grupos formais, e não diferenciam por sexo, nem na interação entre sexo e pertença a grupo, demonstra a importância de incluir no trabalho de promoção e prevenção à saúde adolescente os grupos formais como fatores protetores.

As limitações deste estudo se referem à dificuldade em encontrar estudos anteriores que abordassem a temática da participação dos adolescentes em grupos formais, especialmente para a comparação dos dados encontrados. Da mesma forma, encontraram-se poucos estudos utilizando o Pil-Test no Brasil, dificultando comparações de resultados. A amostra limitou-se a um município da Região Metropolitana de Porto Alegre, não podendo ser generalizada, porém podendo-se pensar que os resultados seriam semelhantes em populações da mesma faixa etária. Sugere-se que novos estudos sejam realizados, no sentido de ampliar a amostra e aprofundar a questão da importância dos grupos formais para a saúde adolescente.

\section{Referências}

Abrantes, J. C. (Coord.) (2002). Os jovens e a internet: representação, utilização, apropriação. Instituto de Estudos Jornalísticos, Faculdade de Letras, Universidade de Coimbra. Obtido em 24 de setembro de 2010 do World Wide Web: 
http://www.bocc.ubi.pt/pag/_texto.php3?html2

=abrantes-jose-carlos-jovensinternet.html.

Adler, N. (1997). Purpose of Life. Obtido em 2 de agosto de 2009 do World Wide Web: http://www.macses.ucsf.edu/Research/Psychoso $\mathrm{cial} /$ notebook/purpose.html.

Adler, N. \& Stewart, J. (2004). Self-Esteem. Obtido em 18 de agosto de 2009 do World Wide Web: http://www.macses.ucsf.edu/Research/Psychoso $\mathrm{cial} /$ notebook/selfesteem.html.

Antunes, C., Sousa, M .C., Carvalho, A., Costa, M., Raimundo, F., Lemos, E., Cardoso, F., Gomes F., Alhais, D., Rocha, A. \& Andrade, A. (2006). Autoestima e comportamentos de saúde e de risco no adolescente: efeitos diferenciais em alunos do $7^{\circ}$ ao $10^{\circ}$ ano. Psicologia, Saúde \& Doenças, 7(1), 117123.

Aquino, T. A. A., Serafim, T. D. B., Silva, H. D. M., Barbosa, E. L., Cirne, E. A., Ferreira, F. R. \& Dantas, P. R. S. (2010). Visões de morte, ansiedade e sentido da vida: um estudo correlacional. Psicologia Argumento, 28(63), 289-302.

Assis, S. G., Avanci, J. Q., Silva, C. M. F. P., Malaquias, J. V., Santos, N. C. \& Oliveira, R. V. C. (2003). A representação social do ser adolescente: um passo decisivo na promoção da saúde. Ciência e Saúde Coletiva, 8(3), 669-679.

Baiocchi, A. C. \& Magalhães, M. (2004). Relações entre processos de comprometimento, entrincheiramento e motivação vital em carreiras profissionais. Revista Brasileira de Orientação Profissional, 5(1), 63-69.

Barros, L. P., Gropo, L. N., Petribú, K. \& Colares, V. (2008). Avaliação da qualidade de vida em adolescentes. Jornal Brasileiro de Psiquiatria, 57(3), 212-217.

Bassen, C. R. \& Lamb, M. E. (2006). Gender differences in adolescents' self-concepts of assertion and affiliation. European Journal of Developmental Psychology, 3(1), 71 - 94.

Borges, L. de O. \& Argolo, J. C. T. (2002). Adaptação e validação de uma escala de bem-estar psicológico para uso em estudos ocupacionais. Avaliação Psicológica, 1, 17-27.

Bulgacov, Y. L. M., Ribeiro, A., Cobalchini, C., Souza, I. \& Diório, Z. (2001). Identidade profissional e projeto de vida: leitura da construção da identidade em adolescentes. Artigo apresentado no XXVIII
Congresso Interamericano de Psicologia. Santiago: Chile.

Costa, A. C. G. (2000). Protagonismo juvenil: adolescência, educação e participação democrática. Salvador: Fundação Odebrecht.

Costa, M. C. O. \& Bigras, M. (2007). Mecanismos pessoais e coletivos de proteção e promoção da qualidade de vida para a infância e adolescência. Ciência \& Saúde Coletiva, 12(5), 1101-1109.

Danielsen, A. G., Samdal, O., Hetland, J. \& Wold, B. (2009). School-related social support and student's perceived life satisfaction. The Journal of Educational Research, 102(4), 303-320.

Feixa, C. (1988). De jóvenes, bandas y tribus. Barcelona: Ariel.

Ferreira, M. A., Alvinir, N. A. T., Teixeira, M. L. O. \& Veloso, R. C. (2007). Saberes de adolescentes: estilo de vida e cuidado à saúde. Texto Contexto Enfermagem, 16(2), 217-24.

Frankl, V. (1987). Em busca de sentido: um psicólogo no campo de concentração. Porto Alegre, RS: Sulina.

Garaigordobil, M. (2009). A comparative analysis of empathy in childhood and adolescence: gender differences and associated socio-emotional variables. International Journal of Psychology and Psychological Therapy, 9(2), 217-235.

Goldbeck, L., Schmitz, T. G., Besier, T. Herschbach, P. \& Henrich, G. (2007). Life satisfaction decreases during adolescence. Quality of Life Research, 16, 969-979.

Harlow, L. L., Newbomb, M. D. \& Bentler, P. M. (1987). Purpose in Life Test assessment using latent variable methods. British Journal of Clinical Psychology, 26, 235-236.

Heilborn, M. L. (2006). Experiência da sexualidade, reprodução e trajetórias biográficas juvenis. Em: M. L., Heilborn, E. M. L. Aquino, M. Bozon \& D. R. Knauth (Org.). O aprendizado da sexualidade: reprodução e trajetórias sociais de jovens brasileiros (pp. 29-57). Rio de Janeiro: Garamond e Fiocruz.

Huebner, E. S., Laughlin, J. E., Ash, C. \& Gilman, R. (1998). Further validation of the Multidimensional Students' Life Satisfaction Scale. Journal of Psychoeducational Assessment, 16, 118-134.

Huebner, E. S., Suldo, S. M., Valois, R. R., Darne, J. W. \& Zullig, K. (2004). Brief Multidimensional Students' Life Satisfaction Scale: sex, race, and 
grade effects for a high school sample. Psychological Reports, 94, 351-356.

IBGE - Instituto Brasileiro de Geografia e Estatística (2009). Estimativas da população para $1^{\circ}$ de julho de 2009. Estimativas de população. Obtido em 14 de dezembro de 2009.

Kell, M. R. (2004). A juventude como sintoma da cultura. Em R. Novaes \& P. Vannuchi (Orgs.). Juventude e sociedade: trabalho, educaşão, cultura $e$ participação (pp. 89-114). São Paulo: Fundação Perseu Abramo.

Klosinski, G. (2006). A adolescência hoje: situações, conflitos e desafios. Rio de Janeiro, RJ: Vozes.

Locatelli, A. C. D., Bzuneck, J. A. \& Guimarães, S. E. R. (2007). A motivação de adolescentes em relação com a perspectiva de tempo futuro. Psicologia: Reflexão e Crítica, 20(2), 268-276.

Ministério da Saúde (1997). Conselho Nacional de Saúde. Diretrizes e normas para pesquisa envolvendo seres bumanos. Resolução CNS 196/196. Brasilia: Ministério da Saúde.

Montemayor, R. (1994). The study of interpersonal relationships during adolescence. Em: R. Montemayor, G. R. Adams \& T. P. Gullota (Orgs.), Personal relationships during adolescence (pp. 16). Advance in Adolescent Development: Thousand Oaks, London, New Delhi: SAGE Publications.

Nascimento, I. P. (2006). Projeto de vida de adolescentes do ensino médio: um estudo psicossocial sobre suas representações. Revista Imaginário, 12(12), 55-80.

Paredes, E. C. \& Pecora, A. R. (2004). Questionando o futuro: as representações sociais de jovens estudantes. Psicologia: Teoria e Prática, 6(n.spe), 4965.

Passareli, P. M. \& Silva, J. A. (2007). Psicologia Positiva e o estudo do bem-estar subjetivo. Estudos de Psicologia Campinas, 24(4), 513-517.

Pavía, V. Gerlero, J. \& Apendino, J. (1995). Adolescencia, grupo y tiempo libre. Buenos Aires: Humanitas.
Romanelli, G. \& Prieto, D. I. C. (2002). Adolescentes do sexo feminino: a família, grupo de pares e relações afetivas. Paidéia: 12(22), 57-68.

Rosenberg, M. (1986). Conceiving the self. Florida: Krieger: Malabar.

Ryff, C. D. (I989). Happiness is everything, or is it? Explorations on the meaning of psychological well-being. Joumal of Personality and Social Psychology, 57, 1069-1081.

Sánchez, A .V. \& Escribano, E. A. (1999). Medição do autoconceito. Bauru, SP: Edusc.

Sarriera, J. C., Schwarcz, C. \& Câmara, S. G. (1996). Bem-estar psicológico: análise fatorial da Escala de Goldberg (GHQ-12) numa amostra de jovens. Psicologia, Reflexão e Crítica, 9(2), 293-306.

Schoen-Ferreira, T. H., Aznar-Farias, M. \& Silvares, E. F. M. (2009). Desenvolvimento da identidade em adolescentes estudantes do ensino médio. Psicologia: Reflexão e Crítica, 22(3), 326-333.

Secretaria da Educação do Rio Grande do Sul (SEC/RS) (2009). Obtido em 19 de julho de 2009 do World Wide Web: http://www.educacao.rs.gov.br/pse/html/educa.j sp.

Sheldon, L. M. \& Bettencourt, B. A. (2002). Psychological need-satisfaction and subjective well being within social groups. British Journal of Social Psychology, 41, 25-38.

Silva, R. A., Horta, B. L., Pontes, L. M., Faria, A. D., Souza, L. D. M., Cruzeiro, A. L, S. \& Pinheiro, R. T. (2007). Bem-estar psicológico e adolescência: fatores associados. Cadernos de Saúde Pública, 23(5), 1113-1118.

Yalom, I. D. (1984). Psicoterapia existencial. Barcelona: Herder.
Recebido em junho de 2010 Reformulado em setembro de 2010 Aprovado em outubro de 2010 
Sobre as autoras:

Cheila de Oliveira Bueno é psicóloga, graduada pela Universidade Luterana do Brasil (ULBRA/Canoas) e bolsista de iniciação científica IC/CNPQ (2008-julho/2010).

Miriam Raquel Wachholz Strelhow é graduanda em Psicologia (ULBRA/Canoas) e bolsista IC/CNPQ.

Sheila Gonçalves Câmara é doutora em Psicologia e mestre em Psicologia Social e da Personalidade pela Pontifícia Universidade Católica do Rio Grande do Sul; professora da graduação no Curso de Psicologia da Universidade Luterana do Brasil/Campus Canoas e do Programa de Pós-Graduação em Saúde Coletiva/ULBRA, professora do Departamento de Psicologia da Universidade Federal de Ciências da Saúde de Porto Alegre (UFCSPA) e bolsista produtividade do CNPq. 